\title{
The Transfer of Rats from a Familiar to a Novel Environment Prolongs the Increase of Extracellular Dopamine Efflux Induced by CCK8 in the Posterior Nucleus Accumbens
}

\author{
Nathalie Ladurelle, Bernard P. Roques, and Valérie Daugé \\ Département de Pharmacochimie Moléculaire et Structurale U 266 INSERM, URA D 1500 CNRS, Université René \\ Descartes, Faculté des Sciences Pharmaceutiques et Biologiques, 75270 Paris Cedex 06, France
}

The effects of cholecystokinin octapeptide (CCK8) on extracellular dopamine (DA) and its metabolites, 3,4-dihydroxyphenylacetic acid and homovanillic acid, were measured in the nucleus accumbens (N. Acc.). The experiments were carried out using in vivo microdialysis in awake rats submitted, or not, to a novel environment, the four-hole box. The exploratory behavior of the animals was studied concurrently in these boxes and in the elevated plus maze. Without CCK8 treatment, the transfer of anImals from thelr home cages to the four-hole box induced a transient increase in DA efflux and also tended to increase its metabolites in the posterior $\mathbf{N}$. Acc. In rats placed in a familiar environment, the administration of 25 pmol of CCK8 in this region immediately enhanced DA release, with levels rapidly returning to normal at the end of the perfusion. In contrast, this treatment produced a longer effect in rats transferred to the four-hole box, since the DA efflux was still increased $80 \mathrm{~min}$ after the removal of CСK8. The intra-accumbal administration of CCK8 induced a hypoexploration in the four-hole box. Moreover, an anxiogenic-like effect of CCK8 was found in the elevated plus maze, only in rats submitted to a novel environment (four-hole box). These data show that (1) the postero-accumbens DA neurons can be activated by environmental changes and (2) that the intensity of the CCK8 effects on extracellular DA levels and on anxiety-like responses seems to depend on the activity of these neurons previous to CCK8 treatment. The prolonged DA release induced by CCK8 in animals placed in a new situation could correspond to a biochemical anticipation preparing them to react when faced by another stimulus.

[Key words: dopamine release, cholecystokinin, environmental change, nucleus accumbens, microdialysis, freely moving rats]

The discovery of the carboxy-terminal sulfated octapcptide of cholecystokinin (CCK8) in mammalian brain (Dockray, 1976) has generated widespread interest on the role of this peptide in

\footnotetext{
Received Aug. 4, 1994; Ievised Oul. 27, 1994; accepled Nuv. 2, 1994.

We thank Dr. A. Beaumont and Dr. J. Viereck for the stylistic revision of the manuscript and C. Dupuis for her secretarial assistance.

Correspondence should be addressed to Prof. B. P. Roques, Département de Pharmacochimie Moléculaire et Structurale, U 266 INSERM, URA D 1500 CNRS, Université René Descartes, Faculté des Sciences Pharmaceutiques et Biologiques, 4 avenue de l'Observatoire, 75270 Paris Cedex 06, France.

Copyright (C) 1995 Society for Neuroscience 0270-6474/95/153118-10\$05.00/0
}

the regulation of various CNS functions. In 1980, Hökfelt et al. reported that a subpopulation of mesencephalic dopamine (DA) neurons also contains CCK. In rodents, cats, primates, and in humans, these mixed cells were shown to be located primarily in the ventral tegmental area (VTA) and in the substantia nigra pars compacta (Hökfelt et al., 1980; Schalling et al., 1990). Their projections terminate in the limbic region, predominantly in the medial and caudal portions of the nucleus accumbens (N. Acc.) and olfactory tubercles (Studler et al., 1981; Hökfelt et al., 1985, 1986). In the last decade, the functional division of the N. Acc. into anterior and posterior parts has been shown by anatomical, biochemical, and behavioral data. The anterior part receives CCK fibers from cortical regions (pyriform cortex, medial prefrontal cortex, etc.) and from limbic structures such as amygdala (Fallon and Seroogy, 1985; Zaborsky et al., 1985), while the innervation of the postero-medial part arises from CCK neurons of the nucleus tractus solitarius (Wang et al., 1992) or the substantia nigra pars compacta and mainly from the mixed CCKDA neurons of the VTA (Hökfelt et al. 1985). The distinction between these two regions has also been shown by release experiments where CCK 8 differentially modulated DA release from the rostral and the caudal DA terminals of the N. Acc.: a decrease and an increase in DA release have, respectively, been found by different techniques in these two regions (Vickroy and Bianchi, 1989; Marshall et al., 1991; Ladurelle et al., 1993). However, it should be noted that some other groups have not found these effects (IIamilton et al., 1984; Voigt and Wang, 1984; Voigt et al., 1985, 1986; Lane et al., 1987; Ruggeri et al., 1987). These discrepancies probably depend on whether the hasal or the $\mathrm{K}^{+}$-evoked DA release was measured by push-pull cannula, microdialysis in awake or anesthetized animals, or in vitro techniques. CCK8 also modifies several rat behaviors, depending on its injection site in the $\mathrm{N}$. Acc. For instance, a rostral injection of CCK 8 has been shown to elicit a decrease in intracranial self-stimulation (De Witte et al., 1987), to attenuate locomotor activation induced by amphetamine or apomorphine (Weiss et al., 1988; Vaccarino and Rankin, 1989) and to reduce the spontaneous alternation behavior of rats (Daugé et al., 1992). On the other hand, most of the studies have shown that CCK8 injected into the postero-median N. Acc. was unable to modify the spontaneous locomotor activity of rats but potentiated the DA- or amphetamine-induced hyperlocomotion (Crawley et al., 1985; Vaccarino and Rankin, 1989). Finally, some studies in rats have shown that some effects of $\mathrm{CCK} 8$ are revealed in paradigms generally conceptualized as producing anxiety-like internal states and/or tendencies to explore a novel environment. 
Thus, Daugé et al. (1989a) have reported that, injected in the pustero-Inedian N. Acc., CCK8 decreased locomotion and rearings measured in the open field only in rats unaccustomed to this environment. Furthermore, in these animals, CCK8 has been shown to decrease the exploration in the four-hole box and to induce an anxiogenic-like effect in the elevated plus-maze through CCK-A receptor stimulation (Daugé et al., 1989b). Interestingly, these latter effects were suppressed by a 6-hydroxydopamine lesion of the DAergic meso-accumbens pathway and by selective D2 but not D1 antagonists (Derrien et al., 1993).

It is now clearly established that the activity of the DA system, notably in the N. Acc., depends on motivational states, strongly suggesting that, in the previous experiments, the behavioral effects of CCK8 were observed only because the DA system was activated by the new situation (fear and/or curiosity induced by the novelty). In a previous study performed in awake rats habituated to their environment, CCK8 perfused in the posterior N. Acc. was shown to increase the DA efflux measured by microdialysis but had no effect on global motor activity (Ladurelle et al., 1993). Therefore, the goal of this study was to investigate the influence of a novel environment on DA release and on behavioral responses in rats before and after CCK8 treatment. With this aim, the release of DA in the posterior N. Acc. was measured by microdialysis in awake rats placed in a novel environment (the four-hole box) and in rats housed in a familiar one (the box in which they were placed $24 \mathrm{hr}$ before experiments). The influence of CCK8, locally perfused in this region, was then studied on both animal behavior and extracellular levels of DA and of its two catabolites, 3,4-dihydroxyphenylacetic acid (DOPAC) and homovanillic acid (HVA). The two doses of the peptide used in this study ( $1 \mathrm{fmol}$ and $25 \mathrm{pmol}$ ) were based on previous studies, showing behavioral and biochemical modifications after injection of CCK8 into the postero-median $\mathrm{N}$. Acc. (Daugé et al., 1989b; Ladurelle et al., 1993). In both cases, the locally injected doses corresponded to the quantities of CCK8 that were calculated to diffuse across the membrane of the microdialysis probes $(\sim 4 \%)$.

\section{Materials and Methods}

\section{Animals}

I'he subjects used throughout this study were male Wistar rats (Depré, France) weighing $200-220 \mathrm{gm}$ at the time of implantation. They were housed in groups of four in a well-ventilated environment under controlled temperature $\left(22 \pm 1^{\circ} \mathrm{C}\right)$ and humidity $(50 \pm 5 \%)$, with food and water made available ad libitum.

\section{Surgery}

A week before use, the rats were sterentaxically implanted under chloral hydrate anesthesia $(400 \mathrm{mg} / \mathrm{kg}$, i.p.). For microdialysis experiments, an unilateral 20 -gauge $(0.9 \mathrm{~mm}$ in external diameter) stainless steel cannula guide was implanted $1.5 \mathrm{~mm}$ above the left side posterior $\mathrm{N}$. $\Lambda \mathrm{cc}$. using coordinates $+1.2 \mathrm{~mm}$ anterior, $1.2 \mathrm{~mm}$ lateral, and $-8 \mathrm{~mm}$ ventral, relative to bregma and skull surface (Atlas of Paxinos and Watson, 1986). For the intra-accumbens injections, rats were bilaterally implanted with stainless steel cannula guides of 25 gauge $(0.6 \mathrm{~mm}$ in diameter) $1.5 \mathrm{~mm}$ above the $\mathrm{N}$. Acc. (anterior, $+1.2 \mathrm{~mm}$; lateral, $\pm 1.2 \mathrm{~mm}$; ventral, $-7.0 \mathrm{~mm}$ ). After the operation, animals received an intramuscular antibiotic injection to prevent infection (Extencilline, $0.3 \mathrm{MU} / \mathrm{kg}$ ).

\section{Injection procedure}

Intra-accumbal injections were made using 30.5 gauge stainless steel needles connected to microsyringes (Hamilton) by polyethylene tubing. CCK8 or vehicle were administered by an infusion pump (Precinorm) under a constant volume $(0.2 \mu \mathrm{l} / \mathrm{side})$ in $2 \mathrm{~min}$. The needles were left in place for $30 \mathrm{sec}$ after the end of injection to allow diffusion of the liquid. After the injection, rats were replaced in their home cages to wait for behavioral testing.

\section{Brain dialysis procedure}

The dialysis probes, constructed according to Robinson and Whishaw (1988), consisted of a $3 \mathrm{~mm}$ long semipermeable cellulose membrane (molecular cut-off $6000 \mathrm{Da}$, outer diameter $0.25 \mathrm{~mm}$; Spectrum, Medical Industries, Inc.) connected to a perfusion system (previously described in Ladurelle et al., 1993). The probes were inserted into the chronically implanted cannula guides until the active membrane was positioned in the N. Acc. and were maintained by a locking screw. This placement was carried out $18-20 \mathrm{hr}$ prior to the start of the experiments and animals were put into individual black boxes $(40 \times 40 \times 40 \mathrm{~cm}$ ). This latency period was required to minimize behavioral and biochemical disturbances due to the probe implantation.

The brain microdialysis procedure consisted of a constant probe perfusion with dialysis buffer $\left(120 \mathrm{~mm} \mathrm{NaCl} ; 5 \mathrm{~mm} \mathrm{KCl} ; 1.8 \mathrm{~mm} \mathrm{CaCl}_{2}\right.$; $1.2 \mathrm{~mm} \mathrm{MgCl} ; 0.2 \mathrm{~mm}$ phosphate-buffered saline; $\mathrm{pH} 7.4)$ at a flow rate of $2 \mu \mathrm{l} / \mathrm{min}$. In order to reach a steady state of the dialysis around the probe membrane, perfusion was carried out for $2 \mathrm{hr}$ before starting the experiments. For the basal efflux determination, three dialysis samples (10 min each) were collected in small tubes containing $40 \mu \mathrm{l}$ of an antioxidant mixture $(10 \mu \mathrm{l}$ of EDTA $0.04 \%$ in $0.1 \mathrm{M}$ perchloric acid plus $30 \mu \mathrm{l}$ of HPLC mobile phase). The animals were then treated for 10 min by carefully replacing the perfusion medium by a CCK8 solution, avoiding the introduction of bubbles in the system. Two $15 \mathrm{~min}$ samples (to take the dead volume of the catheter and the duration of the behavioral test into account) were collected, after which the $10 \mathrm{~min}$ sample collection was continued for $140 \mathrm{~min}$.

\section{Drug and perfusion}

Intra-accumbens dialysis administrations of CCK 8 were made by perfusing the probes for $10 \mathrm{~min}$ with dialysis buffer (for composition, see above) containing sulfated CCK8 at a flow rate of $2 \mu \mathrm{l} / \mathrm{min}$. Sulfated CCK8 (Asp-Tyr $\left(\mathrm{SO}_{3} \mathrm{H}\right.$ )-Met-Gly-Trp-Met-Asp-Phe- $\mathrm{NH}_{2}$ ) was synthesized in the laboratory. For microdialysis experiments, two doses of CCK8 were administered: $25 \mathrm{pmol}$ and $1 \mathrm{fmol}$ (in $20 \mu \mathrm{l}$ of perfusion medium). The global amounts of CCK8 reaching the $\mathrm{N}$. Acc. were estimated by taking into account the passage of the peptide throughout the dialysis membrane. This recovery was determined in vitro at body temperature $\left(37^{\circ} \mathrm{C}\right)$ by perfusing probes placed in a beaker of microdialysis buffer added to CCK8 radiolabeled ${ }^{3} \mathrm{H}$. The recovery of probes was estimated by the following ratio: radioactivity found in the perfusate/radioactivity in the beaker and was about $3-4 \%$ (for more details in the procedure see Ladurelle et al., 1993).

For tests without microdialysis, CCK8 was dissolved in saline $(\mathrm{NaCl}$ $0.9 \%)$ and bilaterally injected at a dose of $25 \mathrm{pmol} / \mathrm{side}(0.2 \mu \mathrm{l} / \mathrm{side}$ in 2 min).

\section{Behavioral stimulation and monitoring}

Procedure associated with the microdialysis collection. Fifteen minutes after the beginning of the intra-accumbens CCK8 perfusion, the animals, housed in a familiar environment (the individual black boxes mentioned above), were carefully transferred into four-hole boxes (Taghzouti et al,, 1985). The length of the perfusion tubing allowed this transfer to be carried out without removing the probes and, therefore, did not perturb the dialysis. The four-hole box consisted of a black square box $(45 \times 45 \times 24 \mathrm{~cm})$ illuminated from the top (200 lux). Fach corner of the hox was extended by a small corridor, at the end of which was drilled a hole (diameter $3.5 \mathrm{~cm}, 4 \mathrm{~cm}$ above floor) extended by a metallic tube (length $8 \mathrm{~cm}$ ) containing a photoelectric detector. During the test, the rats, initially placed in the middle of the arena, cut the photoelectric beams when they visited the holes. The number and the duration of these beam "interruptions" served as a measure of exploratory behavior and were registered on a computer for the first 15 min following the entrance of the animal in the box. To be sure that the effects observed in the groups transferred to the four-hole box were not an artifact from the stress of handling, a microdialysis experiment was done with animal handled or not, transferred or not, and treated or not by CCK8.

Procedure without microdialysis. Fifteen minutes after the intra-accumbens injection, rats were transferred from their home cage to the four-hole box and the same parameters as those cited above were measured for $15 \mathrm{~min}$. Immediately after this test the animals were submitted 


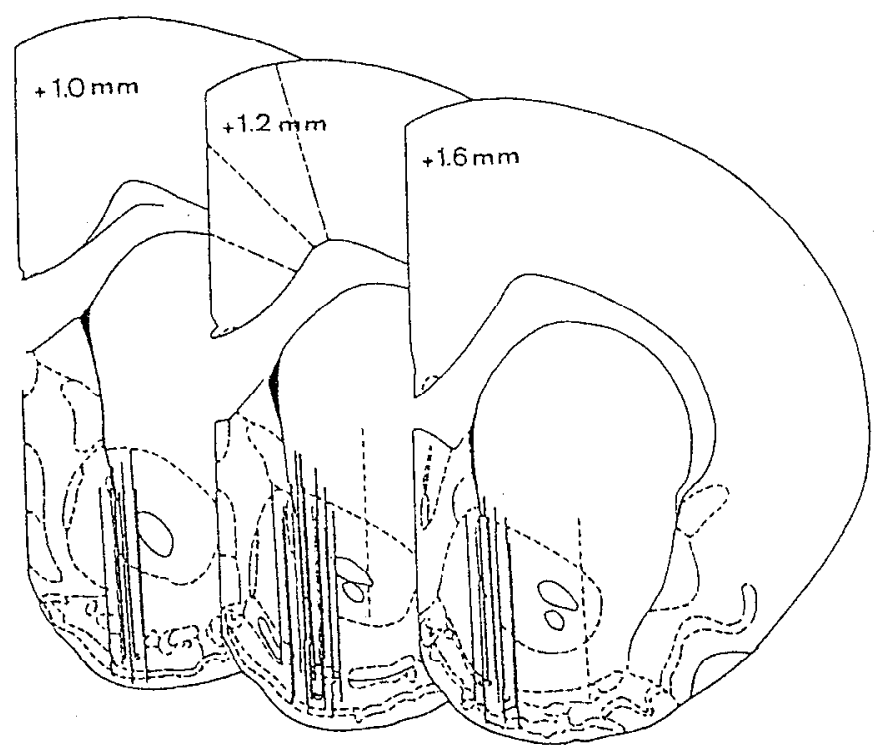

Figure 1. Representation of the histological control of several implantation sites of the dialysis probes. Continuous lines show right positions of the active membrane and broken track lines represent probe positions eliminated from data analysis. The number on each section refers to millimeters rostral to the bregma according to the atlas of Paxinos and Watson (1986).

to another situation: the elevated plus maze. This wooden apparatus consisted of four arms $(50 \times 10 \mathrm{~cm})$ forming a cross with an open square at the intersection of two opposite "closed" arms (C) with walls of $40 \mathrm{~cm}$ height and two opposite "open" arms $(\mathrm{O})$. The maze was elevated $50 \mathrm{~cm}$ above floor and illuminated (300 lux) from the top. At the beginning of the test, the rats were placed facing an open arm in the intersection square. An arm visit was counted when the animals placed all four paws into an arm. The number of entries and the cumulative time spent in the open and the closed arms were measured by an experimenter during a 5 min test session. Another group of animals were identically tested in the elevated plus maze $30 \mathrm{~min}$ after the intracerebral injection but without the previous passage in the four-hole box. The results were expressed as the total $(\mathrm{O}+\mathrm{C})$ number and time spent in the arms and the percentage of the total number or duration of open arms entries over the total visits $(\mathrm{O} / \mathrm{O}+\mathrm{C}$ number and $\mathrm{O} / \mathrm{O}+\mathrm{C}$ duration $)$ were calculated.

\section{HPLC assays}

The levels of DA and its two metabolites, DOPAC and HVA, in the dialysates were determined by electrochemical detection after HPLC separation on a reverse-phase C18 column $(100 \times 2.3 \mathrm{~mm} ; 3 \mu \mathrm{m}$; Catecholamines, Europhor, France). The mobile phase $(0.015 \mathrm{~m}$ sodium acetate; $0.015 \mathrm{M}$ citric acid, $1.2-1.7 \mathrm{~mm}$ sodium octane sulfonate, 0.2 mM EDTA, and $12.5 \%$ methanol, $\mathrm{pH} 3.8$ ), pumped at a flow rate of 0.9 $\mathrm{ml} / \mathrm{min}$, was preoxidized by a guard electrode (5020, ESA) set at 400 $\mathrm{mV}$ in order to minimize background noise, and the three monoamines were assayed by a coulometric detector (Coulochem 5100A, ESA) after oxidation (at $+350 \mathrm{mV})$ and reduction (at $-230 \mathrm{mV}$ ) by a dual analytical cell (5011, ESA). The chromatograms were recorded by a data processor (CR5A, Shimadzu). Peak identification and quantification were carried out by comparison with artificial profiles obtained with standard samples containing from 0 to $2000 \mathrm{pg}$ of DA, DOPAC, and HVA.

\section{Histological control}

At the end of the experiments, an overdose of hydrate chloral was administered to the animals. The brains were removed, frozen, and cut on a microtome and the slices $(50 \mu \mathrm{m})$ were stained with cresyl violet. The position of the probes or needles was determined according to the atlas of Paxinos and Watson (1986). Figure 1 shows the location of some probes implanted in the posterior N. Acc. Probes that traversed more than $70 \%$ of the $\mathrm{N}$. Acc. were considered to be correctly placed. The anterior and lateral positions of the probe were estimated from the island of Calleja and the anterior commissure, respectively. Approximately $7 \%$ of the rats were not correctly implanted and then eliminated from the data calculations. Ninety-three rats were correctly implanted.

\section{Data analysis}

For each rat, the extracellular levels of DA, DOPAC, or HVA were first calculated in picograms released per minute and in nanomolar concentrations, and they were then expressed as the percentage of change from the basal efflux, determined by the average values of the three first samples collected. Then, for each group, the mean and the SEM were calculated. The dialysis time-course data were analyzed using a twoway repeated measure (treatment and time) analysis of variance (ANOVA). If significant effects were observed, a one-way analysis of variance followed by a Dunnett's $t$ test was used to determine the significance at each time point. Moreover, for each experiment, the extracellular levels were also expressed as the percentage of the levels in the control animals before being compared by the same statistical tests. The behavioral data were analyzed by a one-way analysis of variance (ANOVA) followed by a Dunnett's $t$ test for comparison with controls or a Newman-Keuls test for multiple comparisons. The level chosen for statistical significance was $5 \%$.

\section{Results}

Modification of the DA transmission in the posterior N. Acc. Before CCK8 treatment and/or behavioral stimulation, there was no difference in the basal extracellular outflow of DA, DOPAC, and HVA in the posterior N. Acc. of rats between the treated and the saline groups. These levels were about 2.5, 70.0, and $43.0 \mathrm{pg} / \mathrm{min}$ respectively (for more details see Table 1 ).

\section{Table 1. Average basal extracellular levels, uncorrected for recovery, of DA, DOPAC, and HVA in the posterior N. Acc. of rats}

DA

DOPAC

HVA

$$
\begin{aligned}
& 69.3 \pm 7.2(206.1 \pm 21.4) \\
& 71.9 \pm 8.6(213.9 \pm 25.6) \\
& 69.2 \pm 6.9(205.8 \pm 20.5)
\end{aligned}
$$

$73.0 \pm 7.8(217.1 \pm 23.2)$
$71.7 \pm 8.1(213.3 \pm 24.1)$
$72.5 \pm 8.1(215.6 \pm 24.1)$

$$
\begin{aligned}
& 2.6 \pm 0.4(6.9 \pm 1.1) \\
& 2.3 \pm 0.3(6.1 \pm 0.8) \\
& 2.6 \pm 0.4(6.6 \pm 1.1)
\end{aligned}
$$

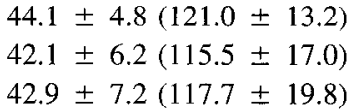

$42.7 \pm 4.9(117.2+13.4)$

$44.1 \pm 5.2(121.0 \pm 14.3)$

$42.6 \pm 4.5(116.9 \pm 12.3)$

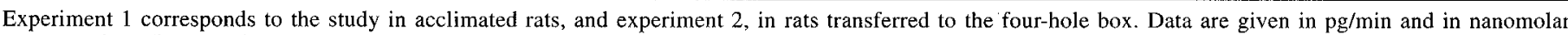

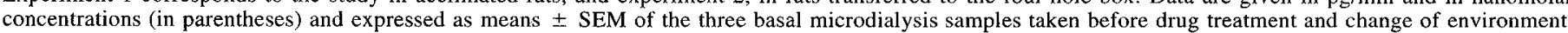

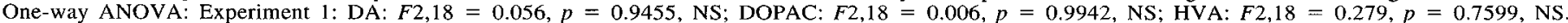
Experiment 2: DA: $F 2,24=0.789, p=0.4657$, NS; DOPAC: $F 2,24=0.040, p=0.9611, \mathrm{NS} ; \mathrm{HVA}: F 2,24=0.024, p=0.9765, \mathrm{NS}$. 
Effects of a novel environment on extracellular DA levels

Without any treatment, the transfer of the animals from their black boxes to the four-hole boxes induced an increase in extracellular DA levels (114.3 $\pm 6.1 \%$ of basal). This effect started as soon as the environment was changed, was maximum for approximately $30 \mathrm{~min}$ following the transfer, and then gradually decreased to basal level even though the rats were still in the box (Fig. 2). Although the metabolite levels also appeared to be modified, the increase in their levels was not significant.

\section{Effects of CCK8 perfusion on rats accustomed to their environment}

Two-way ANOVA showed a significant effect of the CCK8 treatment on extracellular DA levels, which occurred with 25 pmol but not with 1 fmol of the peptide (Fig. 3). Thus, the perfusion of CCK8 in the posterior N. Acc. induced an immediate increase to $145.1 \pm 4.7 \%$ of basal DA levels. After removal of CCK 8 from the perfusion medium, the extracellular DA levels returned rapidly to basal valucs. Despitc a lack of significance, the catabolite levels, especially for DOPAC, were also increased by the treatment and returned more slowly to basal levels.

\section{Effects of CCK8 perfusion on rats placed in a novel environment}

As in previous experiment, the data analysis revealed a significant increase in DA outflow after administration of $25 \mathrm{pmol}$ of CCK8 (Fig. 4). The intensity of this effect was cquivalent to that obtained without changing the rats' environment $(155.5 \pm$ $5.8 \%$ of basal) but its time-course profile was different since the increase persisted for a longer period. However, this prolongation in the effect of CCK8 could only be due to the increase in DA efflux triggered by the novel environment. Therefore, in order to eliminate this parameter, the increase of the extracellular DA levels was expressed in both experiments as the percentage of change compared with the control group (Table 2). This statistical comparison of the effects of CCK8 (25 pmol) showed a significant difference between the animals housed in their boxes and those transferred in the four-hole boxes. For both experiments, the DA increase measured immediately after CCK8 perfusion was of the same order, but from 50 to $140 \mathrm{~min}$ it was higher in animals submitted to the novel environment. Concerning metabolites, DOPAC levels were slightly increased by 25 pmol of CCK8, and HVA also appeared to be increased, but the analysis was not statistically significant.

Figure 2. Effect of the transfer of nontreated rats in the four-hole boxes on extracellular DA $(A)$, DOPAC $(B)$, and HVA $(C)$ levels, uncorrected for recovery, in the posterior $\mathrm{N}$. Acc. The $100 \%$ value was calculated by the mean of three baseline samples taken just before the first time $(10 \mathrm{~min})$ of the curves. The results are expressed as the percentage of this mean (the means are mentioned in Table 1), and bars are SEM. Black squares with thich lines represent the effects on animals $(n=8)$ submitted to the four-hole box; open squares with thin lines show the responses of rats housed in black boxes for $24 \mathrm{hr}(n=9)$. *, $p<0.05$ versus control group; Dunnett's $t$ test. Two-way ANOVA: DA: treatment, $F(1,15)=0.311, p=0.5856$; time, $F(10,150)=1.913$, $p=0.04 / 4$; interaction, $F(10,150)=4.249, p=0.0001$. DOPAC: treatment, $F(1,15)=2.705, p=0.1208$; time, $F(10,150)=1.034, p$ $=0.4179$; interaction, $F(10,150)=1.038, p=0.4148$. HVA: treatment, $F(1,15)=3.260, p=0.0911$; time, $F(10,150)=0.556, p=0.8472$; interaction, $F(10,150)=1.232, p=0.2752$.
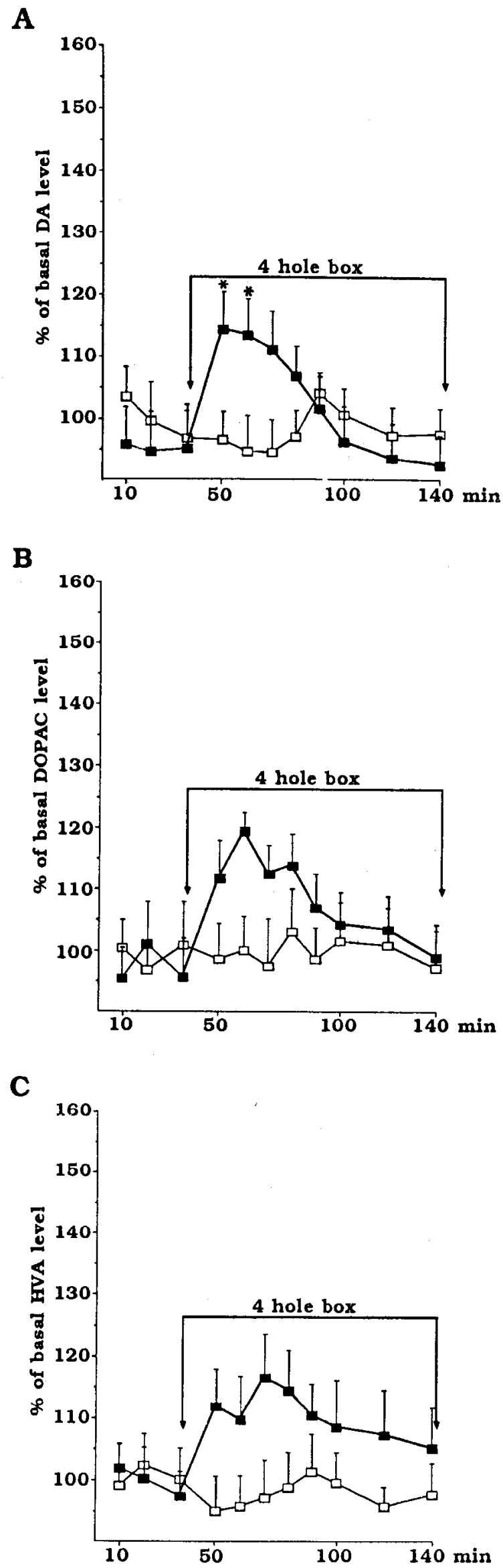


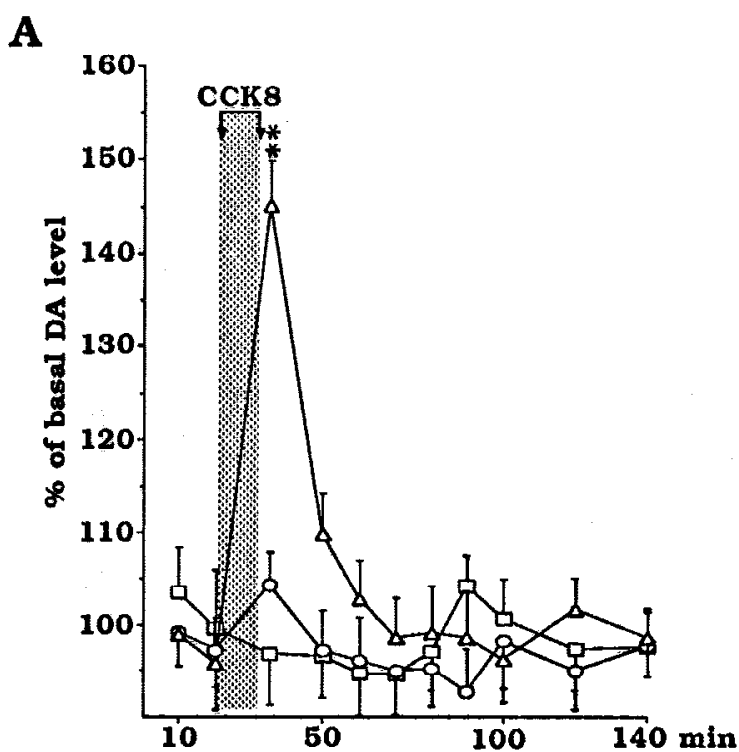

B

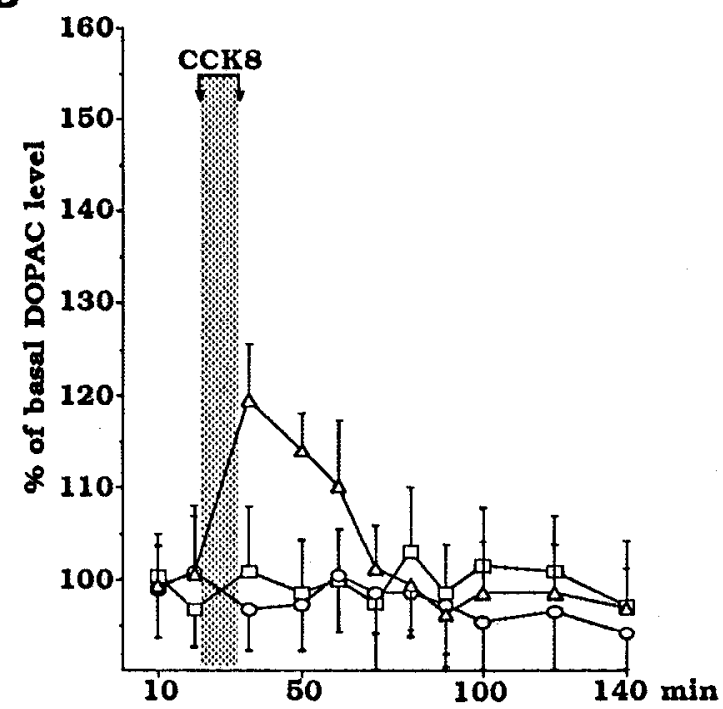

C

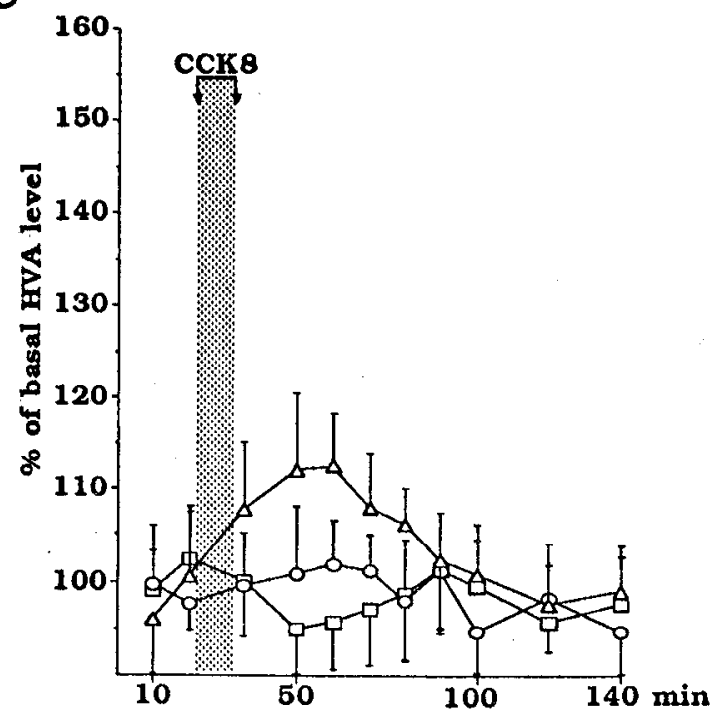

Effect of handling on the DA release induced by CCK8

In order to determine if the prolongation of the DAergic response to $\mathrm{CCK} 8$ in the rats placed in the novel environment really resulted from their exposure to the four-hole box and not from the handling occurring during the transfer, an experiment was done with six groups of animals: saline not handled, saline handled but not transferred, saline transferred, CCK8 (25 pmol) not handled, CCK8 handled but not transferred, and CCK8 transferred. In the groups of rats treated with $\mathrm{CCK} 8$, the statistical analysis of the release of DA in the posterior $N$. Acc. revealed that the rats not handled and those handled but not transferred to the novel environment had the same profile of responses (respective areas under the curves, $14701 \pm 205$ and $14452 \pm 659)$ that were both different from that of rats transferred [area: $18433 \pm 652, F(5,39)=23.753, p=0.0001$ ]. Finally, it should be noted that, even if it was not significant, the handling of control rats induced a very slight increase of the extracellular DA levels as shown by the following areas under the curves: control rats not handled, $11751 \pm 280$; control rats handled, $12035 \pm 531$; and control rats handled and transferred, $12403 \pm 425$.

\section{Behavioral measurements}

Effects of CCK8 perfusion on the rat exploratory activity in the four-hole box. The analysis of variance revealed the presence of behavioral modifications in the rats treated by perfusion of CCK8 during the microdialysis experiment (Fig. 5). Both doses ( 1 fmol and $25 \mathrm{pmol}$ ) produced a hypoexploration, measurable during the $15 \mathrm{~min}$ of the test by a reduction in the number of hole visits $[F(2,18)=6.058, p=0.0097]$. In contrast, the total duration of these visits was not significantly reduced, although it was close to the level of significance $[F(2,18)=2.814, p=$ $0.0864]$.

Effects of CCK8 injection on the rat exploratory activity in the four-hole box (data not shown). One-way ANOVA showed a significant reduction in the numbers of hole visits and a clear tendency of a decrease in visit durations measured over $15 \mathrm{~min}$ in animals locally injected with $25 \mathrm{pmol} / \mathrm{side}$ of CCK8. The number of hole visits passed from $32.3 \pm 4.8$ for saline to 16.5 \pm 3.8 for treated rats $[F(2,18)=6.674, p=0.0187]$ and the duration of visits from $34.3 \pm 5.0$ to $22.0 \pm 5.7$, respectively $[F(2,18)=2.636, p=0.1219]$.

Effects of CCK8 in rats submitted to the elevated plus maze. In saline-treated animals, the passage in the four-holc box before the elevated plus maze test did not modify behavior compared

\section{$\leftarrow$}

Figure 3. Time-course effects of a $10 \mathrm{~min}$ (between 20 and $30 \mathrm{~min}$ of the kinetic study) local perfusion of CCK8 on the extracellular outflow of DA $(A)$, DOPAC $(B)$, and HVA $(C)$, uncorrected for recovery, in the posterior $\mathrm{N}$. Acc. of rats accustomed to their environment. The period of CCK8 infusion in the N. Acc. is shown in the figures by gray areas. For each group of animals, the $100 \%$ value corresponds to the mean value of the first three baseline samples (these means are mentioned in Table 1, experiment 1) and the results are expressed as the percentage of these mean values; bars are SEM. Squares are saline ( $n$ $=9)$, circles are CCK8 $1 \mathrm{fmol}(n=8)$, and triangles are CCK8 25 pmol $(n=10)$. ${ }^{* *}, p<0.01$ versus control group; Dunnett's $t$ test. Two-way ANOVA: DA: treatment, $F(2,24)=3.403, p=0.0500$; time $F(10,240)=6.014, p=0.0001$; interaction, $F(10,240)=4.226, p=$ 0.0001 . DOPAC: treatment, $F(2,24)=0.752, p=0.4824$; time, $F(10,240)=1.253, p=0.2583$; interaction, $F(10,240)=0.967, p=$ 0.5033 . HVA: treatment, $F(2,24)=1.457, p=0.2528$; time, $F(10,240)$ $=0.620, p=0.7959$; interaction, $F(10,240)=0.527, p=0.9536$. 

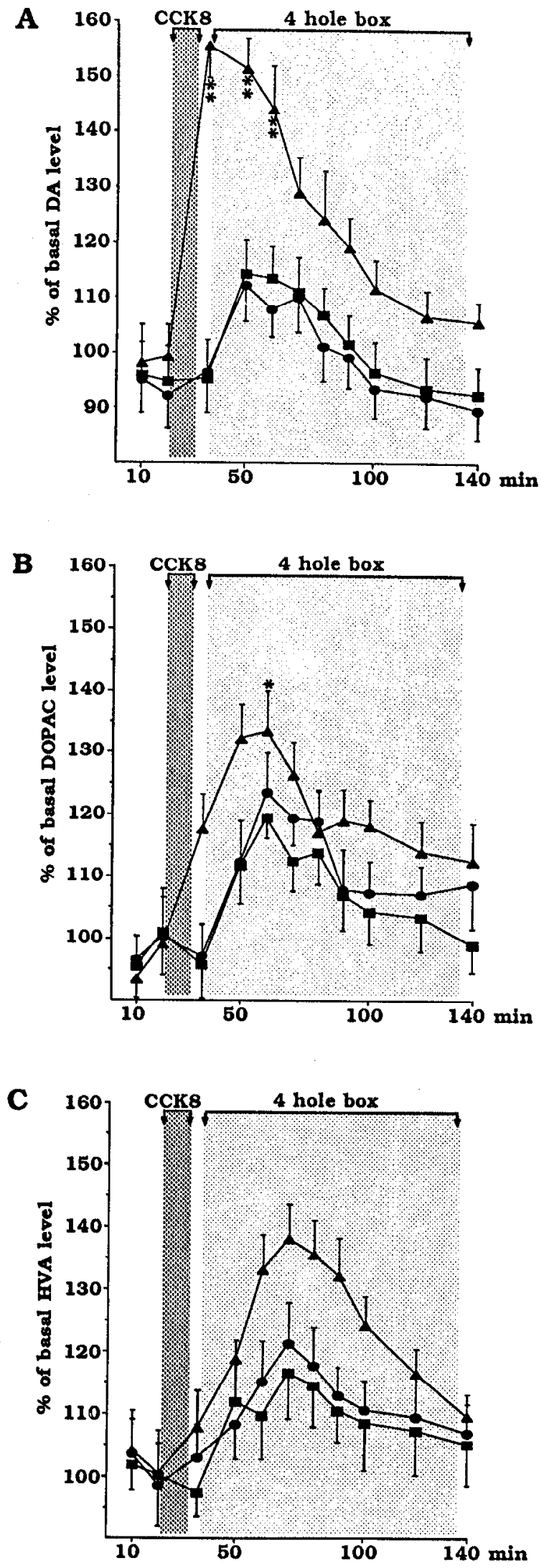

Figure 4. Time-course effects of a $10 \mathrm{~min}$ (between 20 and $30 \mathrm{~min}$ of the kinetic study) local perfusion of CCK8 on the extracellular outflow of DA $(A)$, DOPAC $(B)$, and HVA $(C)$, uncorrected for recovery, in the posterior $\mathrm{N}$. Acc. of rats newly placed in four-hole boxes. The period of CCK8 infusion in the N. Acc. is shown in the figures by dark gray areas and the time spent in the four-hole boxes is represented by to rats directly tested in the maze. In treated animals, CCK8 (25 $\mathrm{pmol} / \mathrm{side}$ ) appeared anxiogenic-like only in rats previously submitted to the four-hole box in which it decreased the percentage of the duration of visits in the open parts $[F(3,35)=4.093, p$ $=0.00137$ ] (Fig. 6). This effect was not the consequence of a general decrease in motor activity since the total number of arm visits $(\sim 8)$ was not significantly changed after CCK8 treatment $[F(3,35)=1.010, p=0.3998]$. However, it is interesting to note that the total duration of arm visits in the treated rats previously placed in the four-hole box was longer than in others animals $[F(3,35)=4.422, p=0.0097$, data not shown]. The statistical analysis revealed that this effect resulted from an increase in the time spent in the closed arms $[F(3,35)=4.097, p=0.0136]$ (Fig. 6).

\section{Discussion}

The purpose of this microdialysis study was to examine concurrently the effects of intra-accumbens administrations of CCK8 on two parameters assumed to be related: behavior and the extracellular DA, DOPAC, and HVA levels in the posterior N. Acc. of awake rats submitted or not to a novel environment. As discussed in the introduction, the two doses $(1 \mathrm{fmol}$ and 25 pmol) of CCK8 used throughout this study were chosen according to previous studies (Daugé et al., 1989b; Ladurelle et al., 1993).

Before testing the CCK8 effects, the first step was to analyze the influence of an environmental change on DA neurotransmission in control rats. The results showed that the transfer of rats to the four-hole boxes slightly increased extracellular DA levels in the posterior N. Acc. (+14\% of basal) and tended also to increase catabolite levels. The modification of the DA release in transferred animals suggests that the novelty has stimulated DA functions. In agreement with this, an increase in DA turnover has previously been shown to occur following the placement of rats in an open field (Tassin et al., 1980). Furthermore, several studies using 6-hydroxydopamine lesions have emphasized the importance of the DA innervation of the N. Acc. in spontaneous and drug-induced exploratory and locomotor behaviors (Fink and Smith, 1980; Taghzouti et al., 1985; CalencoChoukroun et al.,1991; Derrien et al., 1993). Systemic or intraaccumbens administrations of psychostimulant drugs that increase DA release, such as amphetamine, also modify these behaviors (Pijnenburg et al., 1976; Kelley et al., 1986). Taken together, these findings are in good agreement with the proposed functional role of the $\mathrm{N}$. Acc. as an interface between motivation and action (Simon and Le Moal, 1984). Other studies using more stressful situations, such as electroconvulsive shocks (Nomikos et al., 1991) or electric tail shocks (Abercrombie et al., 1989),

$\leftarrow$

pale gray areas. For each group of animals, the $100 \%$ value corresponds to the mean value of the first three baseline samples (these means are mentioned in Table 1, experiment 2), and the results are expressed as the percentage of these mean values; bars are SEM. Squares are saline $(n=8)$, circles are CCK8 $1 \mathrm{fmol}(n=6)$, and triangles are CCK8 25 pmol $(n=7)$. *, $p<0.05 ; * *, p<0.01$ versus control group; Dunnett's $t$ test. Two-way ANOVA: DA: treatment, $F(2,18)=7.612, p=0.0040$; time, $F(10,180)=23.661, p=0.0001$; interaction, $F(20,180)=5.520$, $p=0.0001$. DOPAC: treatment, $F(2,18)=3.463, p=0.0534$; time, $F(10,180)=10.356, p=0.0001$; interaction, $F(20,180)=0.9770, p$ $=0.4921$. HVA: treatment, $F(2,18)=2.243, p=0.1349$; time, $F(10,180)=14.007, p=0.0001$; interaction, $F(20,180)=1.467, p=$ 0.0977 . 

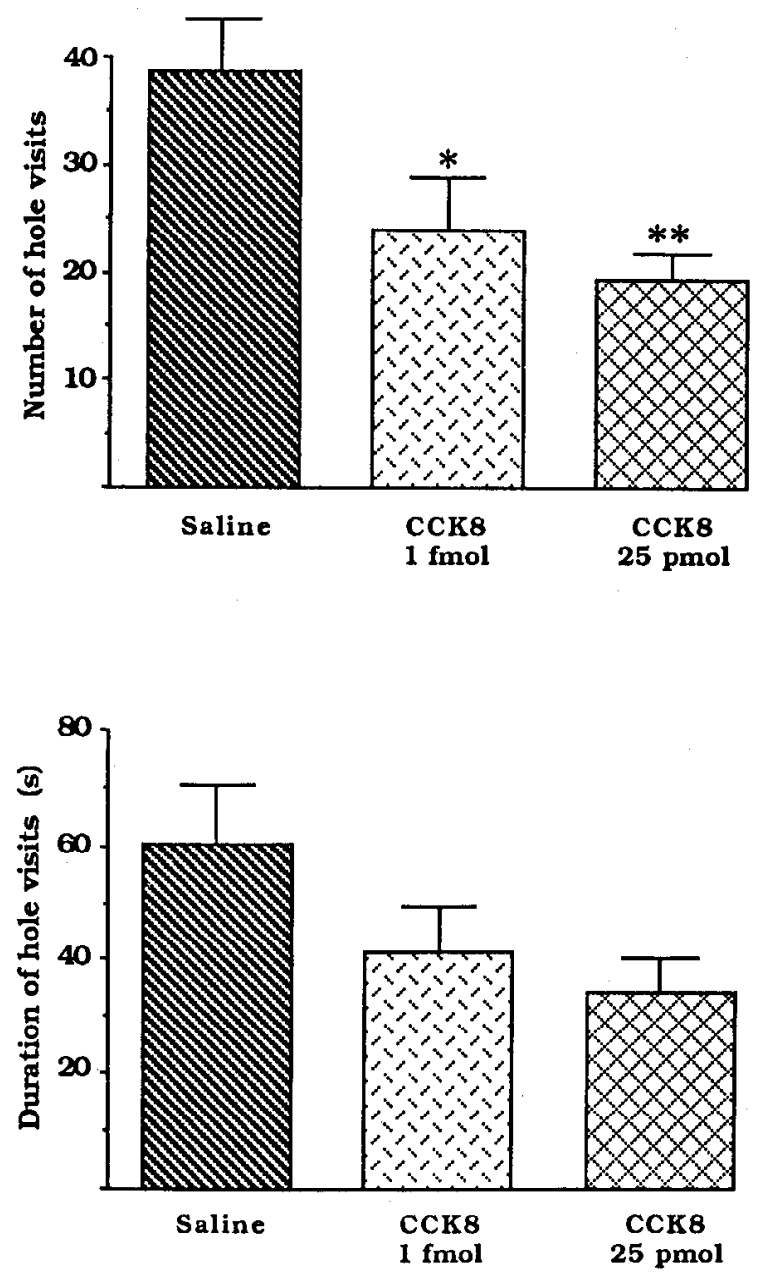

Figure 5. Exploratory behavior over $15 \mathrm{~min}$ in the four-hole box. Upper figure shows the number (means \pm SEM) of hole visits, lower part the duration (means \pm SEM) of these visits. Saline, $n=8$; CCK 8 $1 \mathrm{fmol}, n=6$; CCK8 25 pmol, $n=7 .^{*}, p<0.05 ; * *, p<0.01$ versus control group; Dunnett's $t$ test.

have also shown an increase of extracellular DA efflux in the $\mathrm{N}$. Acc. Interestingly, in these studies, the intensity of the DA increase was two- to threefold higher than that obtained after transfer into the four-hole box. This suggests a direct relationship between the severity of the stress and the strength of the DA response and/or the existence of distinct DA networks differently recruited as function of the experimental situation, as suggested by the results of recent anatomical and pharmacological studies (Zahm, 1989; Heimer et al., 1991; Deutch and Cameron, 1992).

The local perfusion of 25 pmol, but not of 1 fmol, of CCK8 in the posterior N. Acc. of rats induced a large and immediate increase in DA outflow in both animals housed in a familiar environment and those transferred to the four-hole boxes. Interestingly, in hoth cases, these effects were maximal and of the same order of magnitude $(+50 \%$ and $+63 \%$ relative to the saline controls, respectively) in the first sample posttreatment, but they differed in their kinetics. In the first case, the extracellular DA levels returned rapidly to basal levels after interruption of the CCK8 perfusion (Fig. 3A), as previously reported (Ladurelle et al., 1993), while in animals placed in the four-hole boxes, a higher DA efflux was maintained for at least $80 \mathrm{~min}$ (Fig. 4A). This extended effect was not the consequence of the 

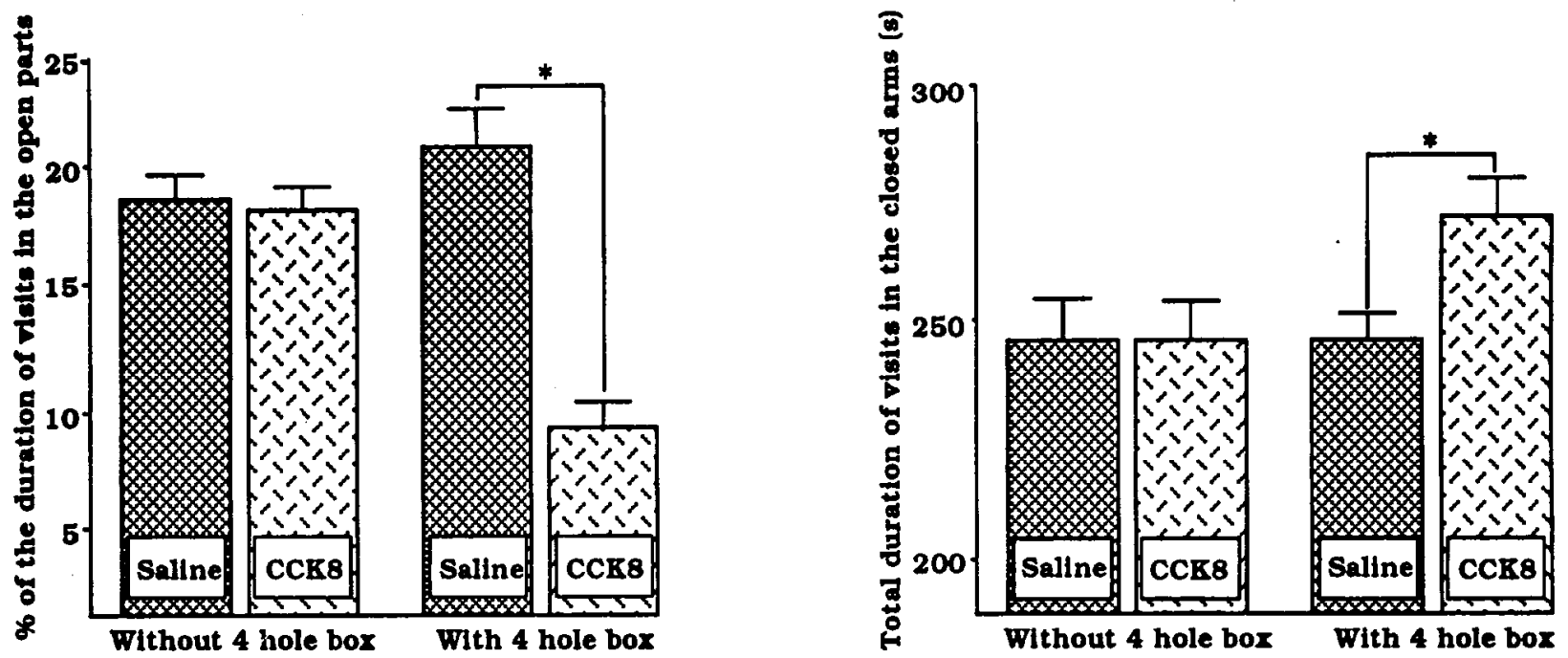

Figure 6. Effect of $25 \mathrm{pmol} / \mathrm{side}$ of CCK8 injected in the posterior N. Acc. of rats in the elevated plus maze. Animals were previously placed or not in the four-hole box. Left panel shows the percentage of the duration of visits in the open parts of the maze and right side the total duration of closed arm visits. Results are expressed in seconds (means \pm SEM). ${ }^{*}, p<0.05$; Newman-Keuls test.

DA increase due to the change of environment (i.e., additive effect) since, when the results were expressed relative to the respective controls (see Table 2), the time-course effects remained significantly higher in the treated animals submitted to the four-hole box. Although there was an increase in catabolites levels in all cases, this was not significant (except for DOPAC in the third sample following the perfusion of 25 pmol of CCK8 in animals submitted to the novel environment). This prolongation of the effect of CCK8 in the transferred animals was not due to the handling of rats occurring during the passage from the familiar box to the four-hole box since in rats handled but not transferred, the increase of the DA release induced by CCK8 had the same duration as in rats not handled. The behavioral analysis showed hypoexploratory effects of both doses of CCK8, illustrated by a reduction in the number of hole visits measured over the first $15 \mathrm{~min}$, following placement of the rats in the fourhole boxes. Even if the experimental conditions for injection and perfusion procedures cannot be exactly the same $(25 \mathrm{pmol}$ of CCK8 in $10 \mathrm{~min}$ for microdialysis perfusions and $25 \mathrm{pmol}$ in 2 min for injections), a similar hypoexploration was found after local injection of $25 \mathrm{pmol} / \mathrm{side}$ of CCK 8 in the posterior $\mathrm{N}$. Acc. of chronically implanted rats showing that, as expected, the experimental conditions of microdialysis did not significantly perturb the rats' behavior. Considering the data reported here, the first interesting observation is the lack of correlation between the doses of CCK8 administered, the induced behavioral effects, and the changes in DA release. Indeed, the hypoexploration obtained with 25 pmol of CCK8 and with the 25,000 -fold lower dose were not significantly different, whereas only the highest dose of CCK8 was associated with a prolonged DA release. This appears to exclude a direct relationship between the biochemical changes and the behavioral responses, despite the fact that the hypoexploration induced by the low dose of CCK $8(1 \mathrm{fmol})$ has been found to be dependent on the integrity of the DA pathway projecting to the posterior N. Acc. and is blocked by the local administration of a D2 receptor antagonist (Derrien et al., 1993). One reason for these apparent discrepancies could be that modification in DA release when the dose of $1 \mathrm{fmol}$ of CCK8 was used was below the sensitivity of the method. Thus, the peptide might have modified extracellular DA levels issuing from a restricted population of neurons, which were not detectable in our experimental conditions, but were sufficient to produce the maximal hypoexploratory effect. Furthermore, in the group of transferred animals, a low DA increase following CCK8 treatment might have been masked by the enhanced DA outflow caused by the environmental change. In contrast, the marked biochemical effect produced by the high dose of CCK8 could result from a strong stimulation of these limited number of neurons and/or from the recruitment of a larger population of neurons. The hypothesis of a specific DA network implied in the exploratory effects studied here seems to be reinforced by the ceiling effect (i.e., the maximal effect), already observed in rats by Daugé et al. (1989a), after injection of $1 \mathrm{fmol}$ to 100 pmol of CCK8 in the posterior N. Acc. A third possibility could be that the behavioral effects obtained with the $1 \mathrm{fmol}$ dose of CCK8 correspond to a modification of the synaptic turnover that is not measurable by microdialysis. Indeed, in 1991, Parsons et al. have shown that a modification of the total amount of DA and its metabolites in the nucleus accumbens can occur without a change of the extracellular levels of DA.

Finally, the most interesting result coming from the present experimental data was the prolonged DA release induced by CCK8 in animals stimulated by a novel environmental situation. It appears that the novelty has made the DA neurons more sensitive to CCK8 stimulation. Interestingly, this prolonged DA release resembles the profile of responses obtained with inhibitors of DA reuptake, such as cocaine or nomifensine (Hurd and Ungerstetd, 1989). Thus, one possible mechanism of action of CCK8 could include an effect at the level of the DAergic terminals. Nevertheless, CCK8 can also indirectly modify the DAergic transmission by an interaction on GABAergic, cholinergic, glutamatergic, or serotonincrgic ncurons or terminals (review in Harro et al., 1993). From a functional point of view, the extended release of DA induced by CCK8 in rats placed in a novel environment could be related to modifications in DA neurons occurring in memory processes. Indeed, an animal confronted with an unknown territory takes some landmarks during the exploratory phase; the memorizing process could be linked 
to the potentialized DA increase. From a behavioral point of view, this prolonged DA release, only observed when the DA neurons have been previously stimulated, could be related to the well-known increase in self-stimulation produced by local administration of CCK8 into the rat posterior N. Acc. (De Witte et al., 1987). The maintenance of increased DA activity might also enable a better adaptation to novel stimuli after the behavioral testing period. Alternatively, it could correspond to a reactional preparation of the neurons in the anticipation of a new situation. This supposition has notably been proposed by Louilot et al. (1986), who showed a maintenance of an increased DOPAC level occurring in a social interaction test (contact with an aggressive intruder), which did not depend on the duration of this behavioral test. This preadaptation, induced by increased DA ncurotransmission, could then be favored by CCK8. Thus, the anxiogenic-like potential displayed by CCK8 after local injection into the N. Acc. in some stressful tests such as elevated plus maze or open-field tests (Daugé et al., 1989a,b) could occur and/or be reinforced exclusively, or at least preferentially, following an activation of DA neurons. In order to test this hypothesis, the anxiogenic-like effects of CCK8 have been measured in the elevated plus maze after injection of the peptide ( 25 $\mathrm{pmol} / \mathrm{side}$ ) in the posterior N. Acc. of rats previously submitted or not to the four-hole box. The experiments showed clearly that the rats "stimulated" by this environment spent less time in the open arms than those coming from their home cages (Fig. 6), supporting the proposition that the anxiogenic-like capacity of CCK8 is, in fact, increased by the change of environment. This is in line with the observation that certain experimental conditions are critical to reveal CCK8 effects (Daugé et al., 1989a) such as preinjection stress conditions in African green monkeys and rodents (review in Harro et al., 1993) or novel environment (Lavigne et al., 1992).

In conclusion, the results presented in this study show that (1) the meso-accumbens DA neurons are activated by novel environmental stimuli and (2) that the effects of CCK8 administered in the posterior N. Acc. on the increased extracellular DA levels seem to depend on the activity of these neurons. The prolonged activation of the mesoaccumbens pathways by CCK8 that occurs in rats facing novel conditions could correspond to reinforced effects and/or preparative actions (a kind of biochemical anticipation). Such a mechanism of action could imply that a disfunctioning of the CCKergic activity in this pathway could lead to some mental disorders such as pathological anxiety, panic disorders, and even drug addiction.

\section{References}

Abercrombie ED, Keefe AK, DiFrischia DS, Zigmond MJ (1989) Differential effect of stress on in vivo dopamine release in striatum, nucleus accumbens and medial frontal cortex. J Neurochem 52:16551658.

Calenco-Choukroun G, Daugé V, Gacel G, Roques BP (1991) Lesion of dopamine mesolimbic neurons blocks behavioral effects induced by the endogenous enkephalins but not by a $\mu$-opioid receptor agonist. Eur J Pharmacol 209:267-271.

Crawley JN, Hommer DW, Skirboll LR (1985) Topographical analysis of nucleus accumbens sites at which cholecystokinin potentiates dopamine-induced hyperlocomotion in the rat brain. Brain Res 335: 337-341.

Daugé V, Dor A, Féger J, Roques BP (1989a) The behavioral effects of CCK8 injected into the medial nucleus accumbens are dependent on the motivational state of the rat. Eur J Pharmacol 163:25-32.

Daugé V, Steimes P, Derrien M, Beau N, Roques BP, Féger J (1989b) CCK8 effects on motivational and emotional states of rat involve
CCK-A receptors of the postero-median part of the nucleus accumbens. Pharmacol Biochem Behav 34:157-163.

Daugé V, Derrien M, Blanchard JC, Roques BP (1992) The selective CCK-B agonist, BC 264 injected in the antero-lateral part of nucleus accumbens, reduces the spontaneous alternation behaviour of rats. Neuropharmacology 31:67-75.

Derrien M, Durieux C, Daugé V, Roques BP (1993) Involvement of $\mathrm{D} 2$ dopamine receptors in the emotional and motivational responses induced by injection of CCK 8 in the posterior part of the nucleus accumbens. Brain Res 617(2):181-188.

Deutch AY, Cameron DS (1992) Pharmacological characterization of dopamine systems in the nucleus accumbens core and shell. Neuroscience 46:49-56.

De Witte P, Heidbreder C, Roques BP, Vanderhaeghen JJ (1987) Opposite effects of cholecystokinin octapeptide (CCK8) and tetrapeptide (CCK4) after injection into the caudal part of the nucleus accumbens or the rostral part and cerebral ventricles. Neurochem Int 10:473 479.

Dockray GJ (1976) Immunochemical evidence of cholecystokinin-like peptides in brain. Nature 264:568-570.

Fallon JH, Seroogy KB (1985) The distribution and some connections of the cholecystokinin neurons in the rat brain. In: Annals of the New York Academy of Science, Vol 448, Neuronal cholecystokinin (Vanderhaeghen JJ, Crawley JN, eds), pp 121-132. New York: New York Academy of Science.

Fink JS, Smith GP (1980) Mesolimbicocortical dopamine terminal fields are necessary for normal locomotor and investigatory exploration in rats. Brain Res 199:359-384.

Hamilton M, Sheehan MJ, De Belleroche J, Herberg LJ (1984) The cholecystokinin analogue caerulein does not modulate dopamine release or dopamine induced locomotor activity in the nucleus accumbens of the rat. Neurosci Lett 44:77-82.

Harro J, Vasar E, Bradwejn J (1993) CCK in animal and human research on anxiety. Trends Pharmacol Sci 14:244-249.

Heimer L, Zahm DS, Churchill L, Kalivas PW, Wohltmann C (1991) Specificity in the projection pattern of accumbal core and shell in the rat. Neuroscience 41:89-125.

Hökfelt T, Skirboll L, Rehfeld JF, Goldstein M, Markey K, Dann O (1980) A subpopulation of mesencephalic dopamine neurones projecting to limbic areas containing a cholecystokinin-like peptide: evidence for immunochemistry combined with retrograde tracing. Neuroscience 5:2093-2194.

Hökfelt T, Skirboll L, Everitt BJ, Meister B, Brownstein H, Jacobs T, Faden A, Kuga S, Goldstein M, Markstein R, Dockray G, Rehfeld J (1985) Distribution of cholecystokinin like immunoreactivity in the nervous system: co-existence with classical neurotransmitters and other neuropeptides. In: Annals of the New York Academy of Science, Vol 448, Neuronal cholccystokinin (Vanderhacghen JJ, Crawley JN, eds), pp 255-274. New York: New York Academy of Science.

Hökfelt T, Holets VR, Staines W, Meister B, Melander T, Schalling M, Schultzberg M, Freedman J, Bjorklund H, Olson L, Lindh B, Elfvin LG, Lundberg JM, Lindgren JA, Samuelsson B, Pernow B, Terenius L, Post C, Everitt B, Goldstein M (1986) In: Progress in brain research, Vol 68, Chap 4 (Hökfelt T, Fuxe K, Pernow B, eds), pp 3370. New York: Elsevier.

Hurd YL, Ungerstedt UI (1989) In vivo neurochemical profile of dopamine uptake inhibitors and releasers in rat caudate-putamen. Eur J Pharmacol 166:251-260.

Kelley AE, Winnock M, Stinus L (1986) Amphetamine, apomorphine and investigatory behavior in the rat: analysis of structure and pattern of responses. Pharmacology 88:66-74

Ladurelle N, Keller G, Koques BP, Daugé V (1993) Effects of CCK8 and of the CCK-B selective agonist BC 264 on extracellular dopamine content in the anterior and posterior nucleus accumbens: a microdialysis study in freely moving rats. Brain Res 628:254-262.

Lane RF, Blaha CD, Phillips G (1987) Cholecystokinin-induced inhibition of DA neurotransmission: comparison with chronic haloperidol treatment. Prog Neuropsychopharmacol Biol Psychiatry 11:291-299.

Lavigne GJ, Millington WR, Mueller GP (1992) The CCK-A and CCK-B receptor antagonists, devazepide and L-365,260 enhance morphine antinociception only in non acclimated rats exposed to a novel environment. Neuropeptides 21:119-129.

Louilot A, Le Moal M, Simon H (1986) Differential reactivity of dopaminergic neurons in the nucleus accumbens in response to different 
behavioral situations. An in vivo voltametric study in free moving rats. Brain Res 397:395-400.

Marshall FH, Barnes S, Hughes J, Woodruff GN, Hunter JC (1991) Cholecystokinin modulates the relcasc of dopamine from the anterior and posterior nucleus accumbens by two different mechanisms. J Neurochem 56:917-922.

Nomikos GG, Ziz AP, Damsma G, Fibiger HC (1991) Effect of chronic electroconvulsive shock on interstitial concentrations of dopamine in the nucleus accumbens. Psychopharmacology (Berlin) 105:230-238.

Parsons. LH, Smith AD, Justice JB Jr (1991) The in vivo microdialysis recovery of dopamine is altered independently of basal level by 6-hydroxydopamine lesions to the nucleus accumbens. J Neurosci Methods 40:139-147.

Paxinos G, Watson C (1986) The rat brain in stereotaxic coordinates, $2 d$ ed. New York: Academic.

Pijnenburg AJJ, Honig WMM, Van der Heyden JAM, Van Rossum JM (1976) Effects of chemical stimulation of the mesolimbic dopamine system upon locomotor activity. Eur J Pharmacol 35:45-48.

Robinson TE, Whishaw IQ (1988) Normalization of extracellular dopamine in the striatum following recovery from a partial unilateral 6-OHDA lesion of the substantia nigra: a microdialysis study in freely moving rats. Brain Res 450:209-224.

Ruggeri M, Ungerstedt U, Agnati LF, Mutt V, Härfstrand A, Fuxe K (1987) Effect of cholecystokinin peptides and neurotensin on dopamine release and metabolism in the rostral and caudal part of the nucleus accumbens using intracerebral dialysis in the anaesthesized rat. Neurochem Int 10:509-520.

Schalling H, Friberg K, Seroogy K, Riederer P, Bird E, Schiffman SN, Mailleux P, Vanderhaeghen JJ, Kuga S, Goldstein M, Kitahama K, Luppi PH, Jouvet M, Hökfelt T (1990) Analysis of expression of cholecystokinin in dopamine cells in the ventral mesencephalon of several species and in humans with schizophrenia. Proc Natl Acad Sci USA 87:8427-8431.

Simon H, Le Moal M (1984) Mesencephalic dopaminergic neurons functional role. In: Catecholamines: neuropharmacology and central nervous system; theoretical aspects, pp 293-307. New York: Liss.

Studler JM, Simon H, Cesselin F, Legrand JC, Glowinski J, Tassin JP (1981) Biochemical investigation of the localization of cholecysto- kinin octapeptide in dopaminergic neurons originating from the ventral tegmental area of the rat. Neuropeptides 2:131-139.

Taghzouti K, Simon H, Louilot A, Herman JP, Le Moal M (1985) Behavioral study after injection of 6-hydroxydopamine into the nucleus accumbens in the rat. Brain Res 344:9-20.

Tassin JP, Hervé D, Blanc G, Glowinski J (1980) Differential effects of a two minutes open field session on dopamine utilization in the frontal cortices of $\mathrm{Balb} / \mathrm{c}$ and $\mathrm{C} 57 \mathrm{BL} / 6$ mice. Neurosci Lett 17:6771.

Vaccarino FJ, Rankin J (1989) Nucleus accumbens cholecystokinin (CCK) can either attenuate or potentiate amphetamine-induced locomotor activity: evidence for rostro-caudal differences in accumbens CCK functions. Behav Neurosci 103:831-836.

Vickroy TW, Bianchi BR (1989) Pharmacological and mechanistic studies of cholecystokinin facilitated $\left[{ }^{3} \mathrm{H}\right]$-dopamine efflux from rat nucleus accumbens. Neuropeptides 13:43-50.

Voigt MM, Wang RY (1984) In vivo release of dopamine in the nucleus accumbens of the rat: modulation by cholecystokinin. Brain Res 296 : 189-194.

Voigt MM, Wang RY, Westfall TC (1985) The effects of cholecystokinin on the in vitro release of newly synthesized $\left[{ }^{3} \mathrm{H}\right]$-dopamine from the nucleus accumbens of rat. J Neurosci 5:2744-2749.

Voigt MM, Wang RY, Westfall TC (1986) Cholecystokinin octapeptides alter the release of endogenous dopamine from the rat nucleus accumbens in vitro. J Pharmacol Exp Ther 237:147-153.

Wang ZJ, Raö ZR, Shi JW (1992) Tyrosine hydroxylase, neurotensine or cholecystokinin containing neurons in the nucleus tractus solitari send projection fibers to the nucleus accumbens in the rat. Brain Res 578:347-350.

Weiss F, Tanzer DJ, Ettenberg A (1988) Opposite actions of CCK8 on amphetamine-induced hyperlocomotion and stereotypy following intracerebroventricular and intra-accumbens injections in rats. Pharmacol Biochem Behav 30:309-317.

Zaborsky L, Alheid GH, Beinfeld MC, Eiden LE, Helmer L, Palkovits $M$ (1985) Cholecystokinin innervation of the ventral striatum: a morphological and radioimmunological study. Neuroscience 14:427-453.

Zahm DS (1989) The ventral striatopallidal parts of the basal ganglia in the rat: compartmentation of ventral pallidal efferents. Neuroscience 30:33-50. 